\title{
Superconductivity versus Diagonal Disorder in the (Hard-Core) Boson-Fermion Model
}

\author{
G. PawŁowski, S. Robaszkiewicz and R. Micnas \\ Institute of Physics, A. Mickiewicz University \\ Umultowska 85, 61-614 Poznań, Poland
}

\begin{abstract}
The influence of randomness of local pair site energies on superconducting properties of a system of coexisting local pairs and itinerant electrons described by the (hard-core) boson-fermion model is analyzed within variational BCS Hartree-Fock approach. Assuming the rectangular distribution function of the random potential the phase diagrams, critical temperatures, and the chemical potential behaviours are determined.
\end{abstract}

PACS numbers: 71.45.Lr, 71.55.Jv, 74.20.-Z

\section{General formulation}

We consider a system of coexisting local electron pairs (LP) and itinerant fermions coupled via charge exchange mechanism, which mutually induces superconductivity in both subsystems. Such a two-component (boson-fermion) model can show features which are intermediate between those of local pair superconductors and those of classical BCS systems and it is of relevance for superconductivity copper oxides and other short coherence length superconductors as well as various bipolaronic and charge ordered materials [1-4]. A similar model has also been adopted for a description of a resonance superfluidity in the Fermi atomic gases with a Feshbach resonance [5].

Here we will analyse the effects of diagonal disorder on the superconducting (SC) $s$-wave phase in such a system.

Recently, this subject has been studied assuming the random LP energy distribution in a bimodal form [6-8]. Here we will present new results obtained for the rectangular distribution function of the random potential and compare them with those obtained for the bimodal distribution. As it will be shown, the detailed 
features of phase diagrams are rather sensitive to the choice of the distribution function. However, an important conclusion concerning a possibility of disorder induced superconductivity is preserved for both types of randomness.

The model Hamiltonian has the form

$$
\begin{aligned}
H= & 2 \sum_{i}\left(\Delta_{0}+E_{i}-\mu\right) n_{i}^{\mathrm{b}}+\sum_{\langle i j\rangle \sigma} t_{i j} c_{i \sigma}^{+} c_{j \sigma}-\mu \sum_{i} n_{i}^{\mathrm{c}} \\
& +I_{0} \sum_{i}\left(c_{i \uparrow}^{+} c_{i \downarrow}^{+} b_{i}+\text { h.c. }\right)
\end{aligned}
$$

$\Delta_{0}$ measures the relative position of the LP level with respect to the bottom of $c$-electron band, $E_{i}$ is the random LP site energy, $I_{0}$ is the intersubsystem charge exchange. The operators for LP (hard-core bosons) $b_{i}^{+}, b_{i}$ obey the Pauli commutation rules [1-3], $n_{i}^{\mathrm{c}}=\sum_{\sigma} c_{i \sigma}^{+} c_{i \sigma}, n_{i}^{\mathrm{b}}=b_{i}^{+} b_{i}, \mu$ is the chemical potential which ensures that a total number of particles per site $n=n_{\mathrm{c}}+2 n_{\mathrm{b}}=\left(\sum_{i}\left\langle n_{i}^{\mathrm{c}}\right\rangle+\right.$ $\left.2 \sum_{i}\left\langle n_{i}^{\mathrm{b}}\right\rangle\right) / N$ is constant.

In analysis of the model we used, as in the case without disorder [1], the mean field Hartree-Fock variational approach (MFA-HFA). First, $F_{0}\left(\left\{E_{i}\right\}\right)$, i.e. the variational free energy for a given fixed configuration of the random site energy $\left\{E_{i}\right\}$ is obtained. Then it is configurationally averaged over the random variable $\left\{E_{i}\right\}$ according to a preset probability distribution $P\left(\left\{E_{i}\right\}\right)$ as $\langle\ldots\rangle_{\mathrm{av}}=\int_{-\infty}^{\infty} \prod_{i} \ldots \mathrm{d} E_{i} P\left(\left\{E_{i}\right\}\right)$. The probability distribution $P\left(\left\{E_{i}\right\}\right)$ of $\left\{E_{i}\right\}$ is assumed to be $P\left(\left\{E_{i}\right\}\right)=\prod_{i} p\left(E_{i}\right)$, with $p\left(E_{i}\right)=p\left(-E_{i}\right)[6-8]$ and for $p\left(E_{i}\right)$ we take the rectangular distribution

$$
p\left(E_{i}\right)=1 /\left(2 E_{0}\right), \quad \text { for } \quad\left|E_{i}\right| \leq E_{0} ; \quad p\left(E_{i}\right)=0, \quad \text { otherwise. }
$$

We have performed a detailed analysis of the phase diagrams and superconducting properties of system considered as a function of $\Delta_{0}, n$, interaction parameters and increasing disorder. For $c$-electrons we have used a model rectangular density of states (DOS) $N(\varepsilon)=1 / 2 D$, for $0<\varepsilon_{k}<2 D, N(\varepsilon)=0$ otherwise, with $2 \mathrm{D}$ being an effective band width.

\section{Summary of results}

In this report we only show selected results concerning the evolutions of the ground state diagrams (Fig. 1), as well as the critical temperatures and chemical potential at $T=0$ (Fig. 2) as a function of increasing disorder for various $n$ and quote the main conclusions.

(1) Similarly as it was for the bimodal distribution of the random potential, also in the present case of rectangular distribution the SC properties of the model are found to be strongly affected by the diagonal disorder in bosonic subsystem (the randomness in the LP site energies). This is in apparent contrast with the one-component conventional $s$-wave BCS-type superconductors, which according to the Anderson theorem are rather insensitive to the diagonal disorder (i.e. to 


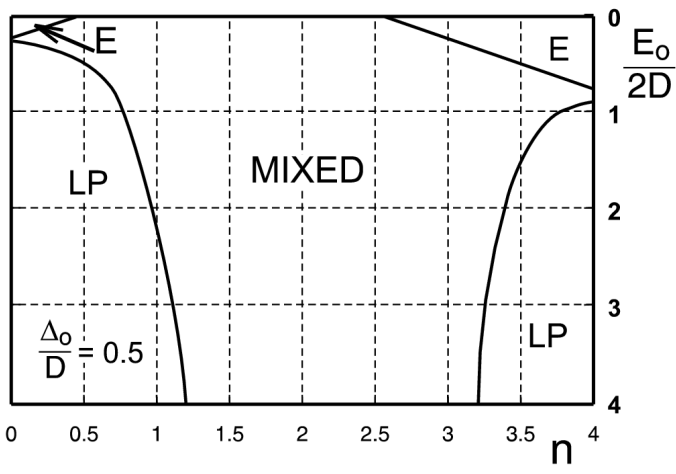

Fig. 1. Ground state diagram of the model (1) as a function of $n=n_{\mathrm{c}}+2 n_{\mathrm{b}}$ and $E_{0} / 2 D(D=z t=4 t)$ for $\left|I_{0}\right| / 2 D=0.1\left(J_{0}=0\right)$ plotted for $\Delta_{0} / D=0.5$. Denotations: LP - "local pair regime" ( $2 n_{\mathrm{b}} \cong n, n_{\mathrm{c}} \cong 0$ for $n<2,2 n_{\mathrm{b}} \cong 2-n, n_{\mathrm{c}} \cong 2$ for $\left.n>2\right)$, E - "c-electrons regime" ( $n_{\mathrm{c}} \cong n, 2 n_{\mathrm{b}} \cong 0$ for $n<2, n_{\mathrm{c}} \cong 2-n, 2 n_{\mathrm{b}} \cong 2$ for $\left.n>2\right)$, MIXED - "mixed regime" $\left(0<n_{\mathrm{c}}, 2 n_{\mathrm{b}}<2\right)$.

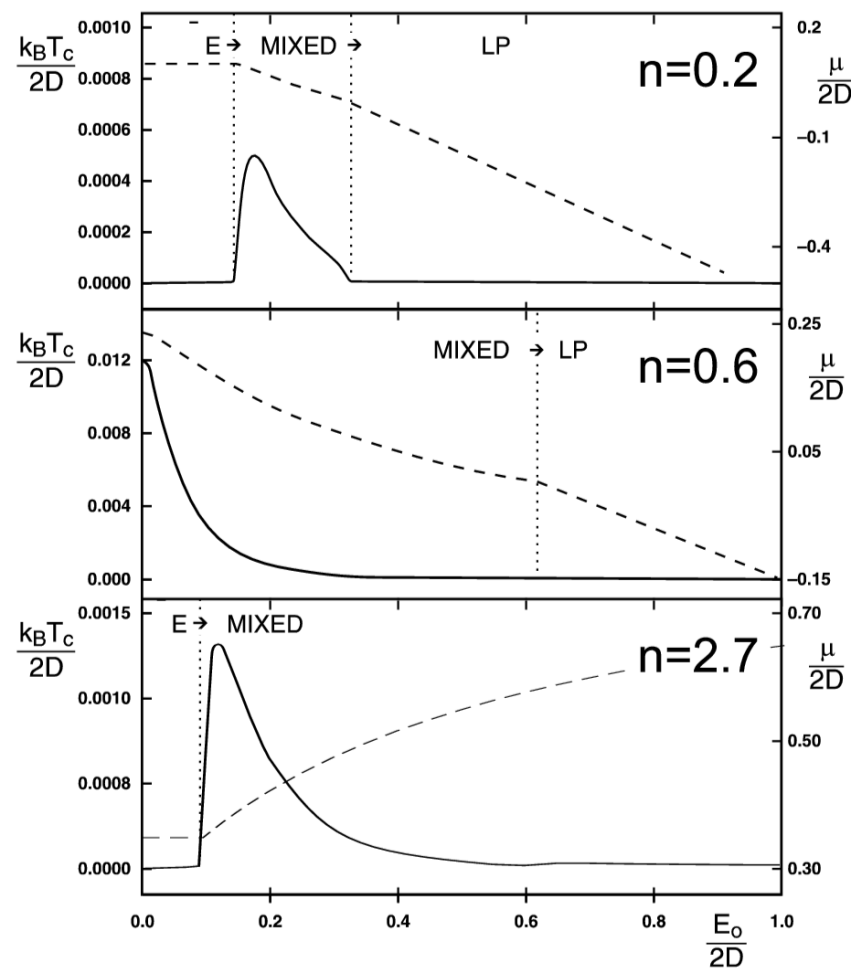

Fig. 2. Evolution of the mean-field critical temperature (solid line) and chemical potential at $T=0$ (dashed line) as a function of increasing disorder $E_{0} / 2 D$ $\left(\Delta_{0} / D=0.5,\left|I_{0}\right| / 2 D=0.1, n=0.2,0.6,2.7\right)$. 
nonmagnetic impurities). The strong dependence of the SC properties on the $E_{i}$ randomness is a combined effect of a change of the effective pairing interaction and the changes in the occupation of fermionic and LP states induced by disorder.

(2) The disorder effects depend in an essential way on the total concentration of carriers $n$ and the position of the LP level $\Delta_{0}$. In general, as for the evolution of the SC properties with increasing disorder, there are several possible types of change-over. In the considered case of rectangular distribution one can single out four types of change-over characteristic of a definite range of $n$. For $\Delta_{0} / D=0.5$ the change-overs are (cf. Figs. 1, 2): (i) $\mathrm{E} \rightarrow \mathrm{MIXED} \rightarrow$ LP, if $n<0.5$; (ii) MIXED $\rightarrow$ LP, if $0.5<n<1.2$; (iii) $\mathrm{E} \rightarrow \mathrm{MIXED}$, if $2.5<n<3.0$; (iv) $\mathrm{E} \rightarrow \mathrm{MIXED} \rightarrow \mathrm{LP}$, if $n>3$ (for denotations see caption of Fig. 1).

(3) There are some qualitative differences between results obtained for the rectangular and the bimodal distribution function of the random potential. In the latter case for a definite range of concentrations one can observe, with increasing $E_{0}$, the change-over from MIXED to the E1 state - "a second c-electrons regime" with $n_{\mathrm{c}} \cong n-1,2 n_{\mathrm{b}} \cong 1$ (it occurs if the bosonic level with the energy $\Delta_{0}-E_{0}$ is completely occupied $2 n_{\mathrm{b}} \cong 1$ since it lies below the Fermi level: $\mu>\Delta_{0}-E_{0}$, whereas the level with the energy $\Delta_{0}+E_{0}$ is empty: $\left.\mu>\Delta_{0}+E_{0}\right)$. In that regime, which is absent in the case of rectangular $E_{i}$ distribution, the critical temperature is very strongly suppressed by disorder and the gap to $T_{\mathrm{c}}$ ratio is shifted towards the BCS value [8].

(4) For both types of $E_{i}$ distribution, in an extended range of $\left\{n, \Delta_{0} / D\right\}$ even a relatively small disorder has strong detrimental effect on $T_{\mathrm{c}}$ (see e.g. the case $n=0.6$ in Fig. 2 and Figs. 2, 3 in Ref. [8]). However there also exist a definite range of parameters $\left\{n, \Delta_{0} / D\right\}$, where the system exhibits a behaviour which can be called a disorder induced superconductivity. At $\Delta_{0} / D=0.5$ this can be observed for $0<n<0.5$ as well as for $n>2.5$ (Figs. 1, 2), i.e. for the concentrations where the change-overs $\mathrm{E} \rightarrow \mathrm{MIXED}$ are realized. In these cases, for $E_{0}=0$ the system is in $E$ regime with $T_{\mathrm{c}} \approx 0$. With increasing disorder, at some nonzero value of $E_{0} / 2 D$, dependent on $\left\{n, \Delta_{0} / D\right\}$ one observes an abrupt increase in $T_{\mathrm{c}}$, the superconducting order parameters and the energy gap, connected with the $\mathrm{E} \rightarrow$ MIXED change-over. With a further increase in $E_{0} / 2 D$ the $T_{\mathrm{c}}$ goes through a round maximum and then it decreases.

(5) Other unique feature of the model analyzed is a possibility of a disorder induced bound pair formation of itinerant electrons. Such phenomena can be observed for the ranges of $\left\{n, \Delta_{0} / D\right\}$, where increasing disorder yields either a sequence of change-overs: $\mathrm{E} \rightarrow \mathrm{MIXED} \rightarrow$ LP or a single change-over: MIXED $\rightarrow$ LP. In both these cases the bound pair formation will occur close to the MIXED/LP boundary (cf. Figs. 1, 2), at which increasing $E_{0}$ places the chemical potential either below the bottom of the $c$-electron band or above the top of the band. 


\section{Acknowledgments}

This work was supported in part by the State Committee for Scientific Research, grant No. 1 P03B 084 26; 2004-2006, and by the Foundation for Polish Science.

\section{References}

[1] S. Robaszkiewicz, R. Micnas, J. Ranninger, Phys. Rev. B 36, 180 (1987); J. Ranninger, S. Robaszkiewicz, Physica B 135, 468 (1985).

[2] R. Micnas, J. Ranninger, S. Robaszkiewicz, Rev. Mod. Phys. 62, 113 (1990) and references therein.

[3] R. Micnas, S. Robaszkiewicz, in: High-T $T_{c}$ Superconductivity 1996: Ten Years after the Discovery, Eds. E. Kaldis, E. Liarokapis, K.A. Müller, NATO ASI Series E, Vol. 342, Kluwer Academic Publishers, The Netherlands 1997, p. 31 and references therein.

[4] R. Micnas, S. Robaszkiewicz, A. Bussmann-Holder, Phys. Rev. B 66, 104516 (2002).

[5] See e.g. Y. Ohashi, A. Griffin, Phys. Rev. Lett. 89, 130402 (2002).

[6] G. Pawłowski, S. Robaszkiewicz, Mol. Phys. Rep. 34/1, 76 (2001); Phys. Status Solidi B 236, 400 (2003).

[7] T. Domański, K.I. Wysokiński, Phys. Rev. B 66, 064517 (2002).

[8] G. Pawłowski, S. Robaszkiewicz, R. Micnas, J. Supercond. 17, 33 (2004). 